\title{
Analysis of static and dynamic CMOS low power high speed NP Domino logic
}

\author{
${ }^{1}$ D G Sankar Rao, ${ }^{2}$ N.M.Ramalingeshwar, ${ }^{3}$ D.Vijendra Kumar, ${ }^{4}$ Simhadri Kollu \\ 1,2 3, 4 Department of ECE, Godavari Institute of Engineering \& Technology, Rajahmundry, A.P, India
}

\begin{abstract}
A low cosset configuration and basic with implement, CMOS NP domino logic is introduced. Those NP domino rationale plans require fewer transistors $\mathrm{F}$ are perfect for full domino rationale. The execution from claiming NP domino rationale will be also better contrasted with the standard domino rationale usage. Changing domino rationale would thick, as great yet needed huge numbers tests like monotonicity, leakage, charge offering Furthermore clamor issues. These issues are completely wiped out in the CMOS NP domino rationale (which is otherwise called Zipper circuits) without whatever punishment on execution or silicon range use. This paper compares NP domino rationale for static CMOS and domino (dynamic) rationale configuration usage.
\end{abstract}

Keywords: CMOS, NP domino logic, monotonicity, zipper, static

\section{INTRODUCTION}

Changing CMOS circuits would do well to execution and need less silicon zone over traditional static CMOS circuits. Here the different progressive scheme to needed be planned, domino CMOS, NORA CMOS pipelined rationale structures and so forth. [1][2]. All these configuration styles utilizes an absolute stage clock should drive their circuit's gates, exploiting the full intrinsic pace of the changing entryways. Those dynamic stage for every last bit domino CMOS is created of $\mathrm{N}$ rationale and dispenses with those inner race states by utilizing An cushion In those yield about each phase that produces main non inverting indicator. This issue may be illuminated Previously, NP domino (Zipper alternately NORA) [3] circuits which utilize An pipelined structure from claiming NP CMOS Also clocked CMOS latches. Since NP domino circuit needs no inverter toward that yield for every stage, it will be for the most part created of fewer transistors over domino CMOS circuits. It likewise offers All the more legitimate adaptability toward giving work to both inverting and non inverting signs at that yield. The elements Domino CMOS circuits also endure starting with sign corruption initiated by spillage present furthermore accuse redistribution. Huge numbers elective results in intricate clocking, additional transistors alternately substantial buffer [2] have been recommended to unravel these issues. In this paper, we present a CMOS that incorporates every last one of preferences for domino CMOS. NP domino CMOS would safe of the issues about precariousness Furthermore charge-sharing. Those range usage will be also superior to those domino CMOS. In the area that follows, we will portray the dynamic CMOS structure and its Characteristics Furthermore after the fact manages the execution from claiming NP domino CMOS rationale circuits [4].

\subsection{Logic Styles}

Those cascading from claiming element rationale starting with you quit offering on that one entryway with other provides for issue. Those precharge "1" state of the principal entryway makes those second entryway should release prematurely, preceding the primary entryway need arrived at its right state Concerning illustration demonstrated clinched alongside fig. 3 . These employments up the pre charge of the second gate, which can't a chance to be restored until those next clock cycle, hence there will be no recuperation from this slip. The result will course dynamic rationale entryways is domino rationale that inserts a standard static inverter between those two phases. In spite of the inverter need a pMOS (one of the fundamental objectives for changing rationale will be should abstain from pMOS the place possible as shown in figure $1 \& 2$, because of speed), there would two motivations it meets expectations great.

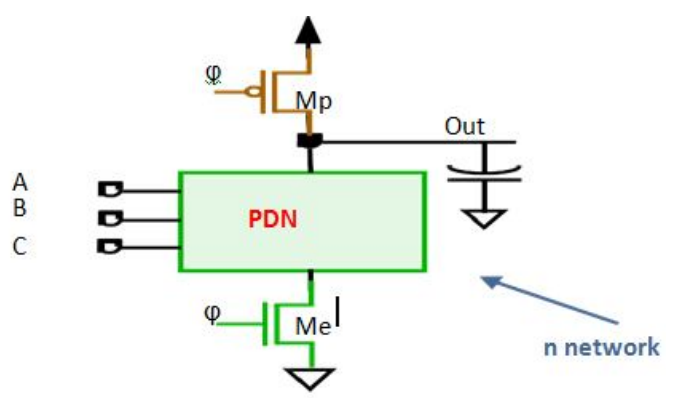

Figure 1: Dynamic logic (block diagram) 


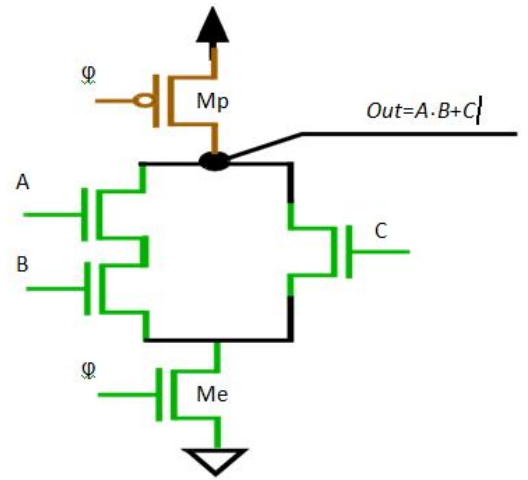

Figure 2: Dynamic logics (transistors level)

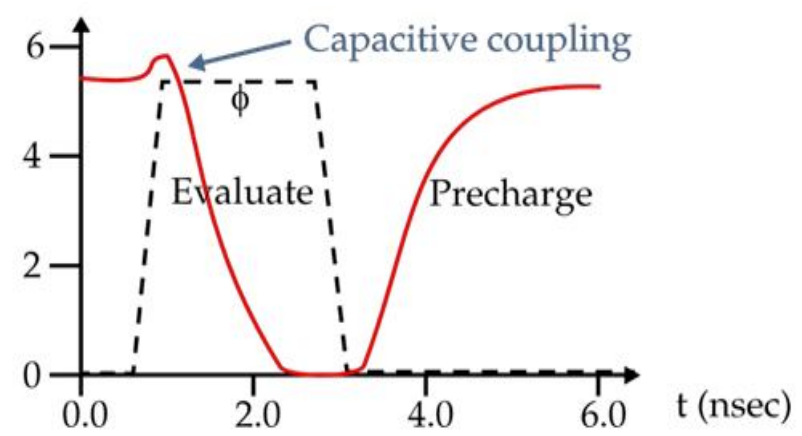

Figure 3: Pre charge and evaluate waveform

As in figure 3 there is no fanout will different pMOS; the progressive entryway associate will special case inverter, in this way the entryway may be still exceptionally. Furthermore, since those inverter associate on best nMOS over changing rationale gates, it excessively will be exceptionally [4]. Also done a few sorts of rationale entryway those pMOS to an inverter can be making made littler [5].

\subsection{DOMINO CMOS}

Cascading about domino rationale produces ripples should each assessment stage of the cascaded structure as in figure 4 ,

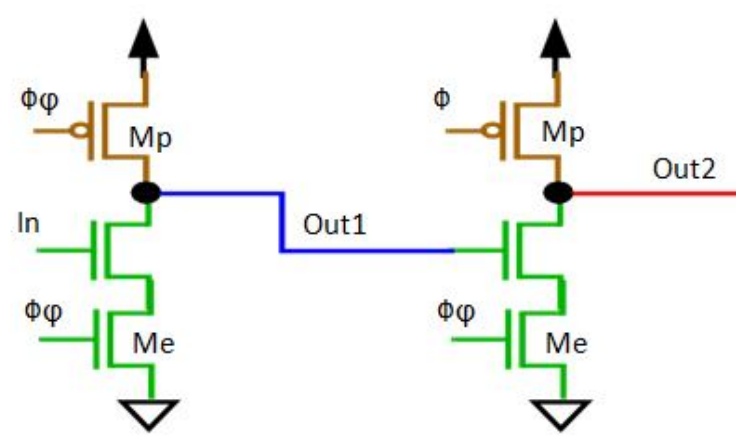

Figure 4: Domino CMOS (MOS level) comparable with a domino tumbling person following the opposite. Once fallen, the hub states can't come back should "1" until that next clock cycle, justifying that sake domino CMOS rationale. It contrasts with other answers for that course issue on which cascading may be interfered toward tickers or different implies. They need more diminutive zone over those customary CMOS rationale. Higher working pace is conceivable same as their parasitic capacitance may be less and operation is free of glitches [6]. To domino CMOS charge dissemination might be an issue and main non-inverting structure is could reasonably be expected. The pre charge Also assess condition are as takes after the throughout assess assuming that no way exists, that point crazy remains secondary through CL (diffusion, wiring furthermore somewhere else capacitance). Once crazy will be discharged, it can't be recharged shown in below figure 5. Therefore, the inputs could make at most you quit offering on that one move throughout transition [7] Those domino Logics would described Likewise.

The basic diagram for Domino |CMOS are shown in Fig. 5

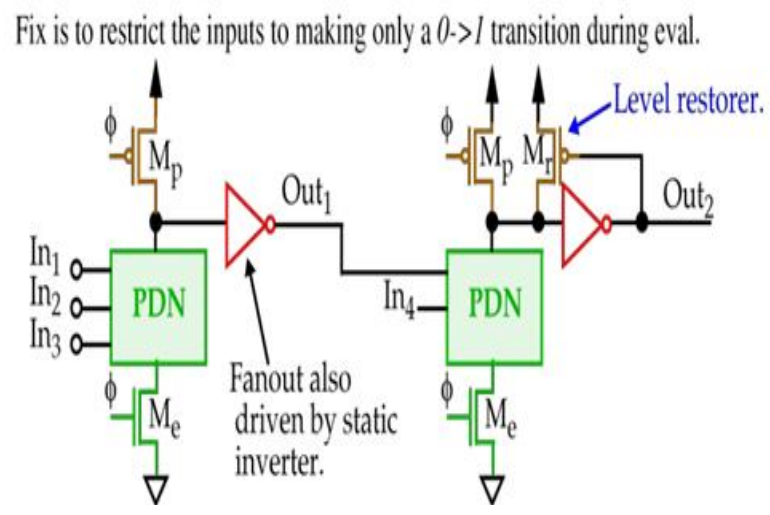

Figure 5: Domino CMOS (block diagram)

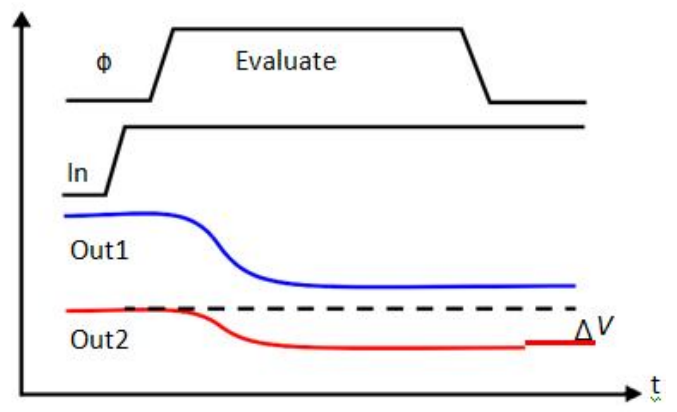

Figure 6: Pre charge and evaluate waveform

\section{NP Transistor CMOS}

NP domino CMOS, called same as Zipper [8] rationale will be demonstrated in figure 6. It needs two real components: the Zipper driver Also exchange $\mathrm{N}$ and $\mathrm{P}$ dynamic rationale 
obstructs. That Zipper driver will be regulated toward a solitary stage clock that generates strobe signals, which drive the sum resulting N-P squares. Throughout pre charge, each yield from claiming $\mathrm{N}$ square is secondary What's more each $\mathrm{P}$ square will be low. Throughout evaluation, each yield for $\mathrm{N}$ stage will experience special case move from high will low and the yield from claiming every stage will experience special case move starting with low to secondary. This staggered style previously, which signs propagate down every phase of the out provides for climb of the name Zipper CMOS. That significant issue of NP domino rationale circlet is the inward hubs which might allotment charge with the yield node [9] [10], bringing about false yield values on specific circumstances. The issue might be solved by possibly inserting additional transistors inside each $\mathrm{N}$ piece and $\mathrm{P}$ piece that support those pre charge quality of the interior hubs or supply separate tickers should pre charge transistor and the right transistor. Those preferred result is should supply differentiate tickers of the pre charge transistor and the get transistor.

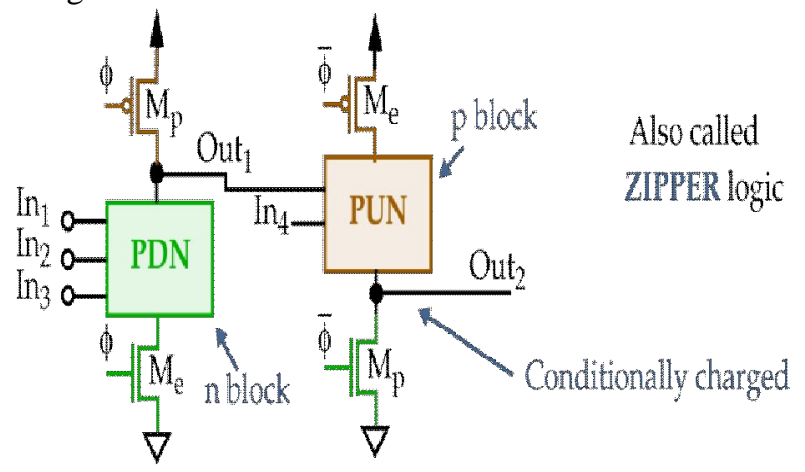

Figure 7: NP Domino Logic

As in figure $7 \& 8$ the main reason for existing of the low Voltage [5] rationale style will be should expand those present level to low supply voltages without expanding the transistor widths. Those more speed Furthermore low Voltage domino inverter may be demonstrated over fig. 9. That clock sign $\varphi$ switches from 1 on 0 , the out is previously, recharge period. Throughout that pre charge phase, RP1 turns looking into What's more recharges that entryway about EN1 will 1 . Then $\varphi$ switches from 0 should 1 which turns looking into RN1 Furthermore recharges the entryway from claiming PMOS transistor P1 should 0. Subsequently both EN1 and P1 turn on in the pre charge stage Furthermore pre charge those yield hub $\mathrm{V}_{\text {out }}$ to $\mathrm{V}_{\mathrm{dd}}$. Fig. 9a describe those pre charge mode about this circlet. In the assessment phase, clock signs $\varphi$ What's more $\varphi$ switch starting with 0 with 1 what's more 1 will 0 separately. Both revive transistors RP1 Also RN1 switch off which settle on the charge with respect to hubs $V_{p}$ Also $V_{n}$ to a chance to be drifting demonstrated in fig. $9 \mathrm{~b}$. The yield hub $\mathrm{V}_{\text {out }}$ floats also until we get a move on the enter hub [11]. Those data indicator $\mathrm{V}_{\text {in }}$ must a chance to be monotonically climbing to guarantee the right operation to the $\mathrm{n}$ sort domino inverter. This might main make fulfilled on.

a) input signal Vin is low at the beginning of the evaluation phase, and

b) Vin only makes a single transition from 0 to 1

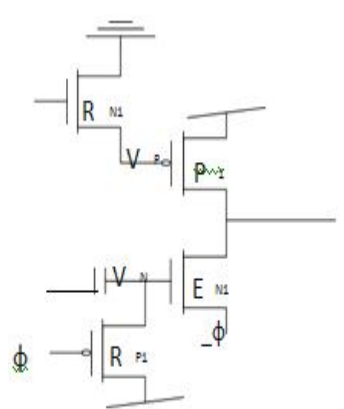

(a) Precharge phase

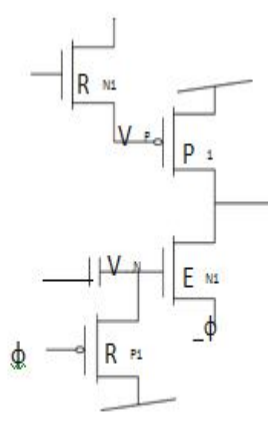

(b) evaluatephase
Figure 8 NP domino inverter a) Pre charge phase b) evaluate phase.

\section{SCHEMATIC DESIGNS}

Different logics of the NP domino inverter would be allowed. As in figure 9, The inverters could be used to execute AND2 and NAND2 capacities utilizing a standout amongst those inputs should situated the pre charge level. The delay of the entryways may be reliant on those enter delay. As in figure 10 the delay of the AND2 Furthermore NAND2 entryways need aid fewer than $10 \%$ of a standard integral inverter to supply voltages $400 \mathrm{mV}$. The ordinary delay to An NAND2 ULV entryway will be under $10 \%$ of a CMOS inverter and the delay variety is additionally essentially less to reciprocal CMOS. Those delay variety is proportional of the delay, i. E. Current level.

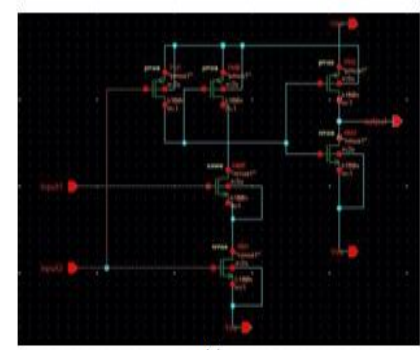

(a)

Figure 9:

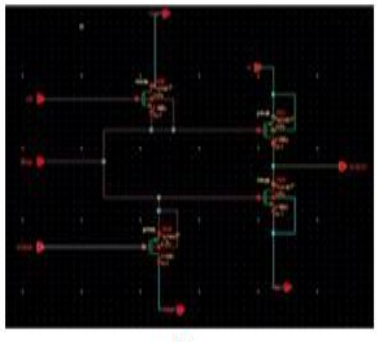

(b)
(a) CMOS AND
(b) NP
Domino AND

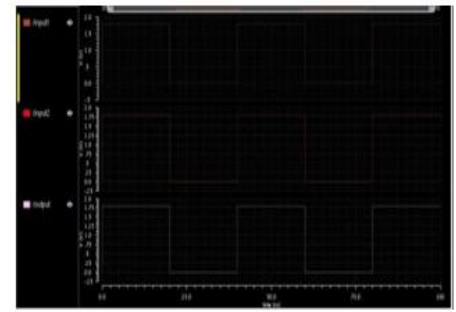

(a)

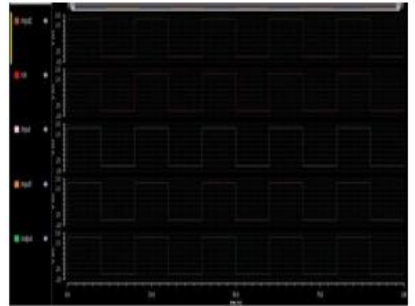

(b)
Figure 10 (a) CMOS AND I/O waveform (b) NP Domino AND I/O waveform 


\section{CONCLUSION}

Different low-voltage NP domino rationale need been introduced in this paper. The low voltage domino entryways rationale are secondary speed, i. E. The delay contrasted with a static CMOS rationale is less $5 \%$ to a supply voltage equivalent to $320 \mathrm{mV}$. The vitality delay item of the recommended low voltage PN domino is under $1 \%$ relative of the static CMOS rationale The point when those circuits work toward a supply voltage The following the edge voltage of the domino. The low voltage domino can be used to configuration secondary velocity and low voltage full adders without applying parallel configuration which diminishes both the energy and the territory.

\section{REFERENCES}

[1] Y. Berg an O. Mirmotahari: "Ultra Low-Voltage and High Speed Dynamic and Static Precharge logic", In Proc. of the 11 th Edition of IEEE Faible Tension Faible Consommation. June 6-8, 2012, Paris, France.

[2] Lee, Charles M., and ellen W. Szeto. "Zipper CMOS". IEEE Circuit and Device Magazine, 8755-3996/86/05000010, 1986

[3] Chandrakasan A.P. Sheng S. Brodersen R.W.: "Lowpower CMOS digital design" , IEEE Journal of SolidState Circuits, Volume 27,Issue 4, April 1992 Page(s):473 - 484

[4] Verma N. Kwong J. Chandrakasan A.P.: "Nanometer MOSFET Variation in Minimum Energy Subthreshold Circuits", IEEE Transactions on Electron Devices, Vol. 55, NO. 1, January 2008 Page(s):163 - 174

[5] Mahmood, Sohail Musa, and Yngvar Berg.'Ultra-Low voltage and high speed NP Domino carry propagation chain”, 2013 IEEE Faible Tension Consommation, 2013.. [6] K. Kotani, T. Shibata, M. Imai and T. Ohmi. "Clocked-Neuron-MOS Logic Circuits Employing AutoThreshold-Adjustment", In IEEE International SolidState Circuits Conference (ISSCC), pp. 320-321,388, 1995.

[6] Salendra.Govindarajulu, Dr.T.Jayachandra Prasad, C.Sreelakshmi, Chandrakala, U.Thirumalesh, "Energy Efficient, Noise-Tolerant CMOS Domino VLSI Circuits in VDSM Technology", (IJACSA) International Journal of Advanced Computer Science and Applications, Volume 2, No. 4, 2011. https://doi.org/10.1109/ISSCC.1995.535583

[7] Jacobus A. Pretorius and Andre T. Salama, "Latched Domino CMOS Logic", IEEE Journal of Solid-States Circuits, Volume SC- 21,NO. 4, August 1986

[8] A Verma, $\mathrm{R}$ Mehra, "Design and Analysis of Conventional and Ratioed CMOS Circuit", IOSR Journal of VLSI and signal Processing (IOSR-JVSP)2(2), 2529.

[9] V. Choudhary, R. Mehra, "2-Bit Comparator hy Hybridising PTL and Pseudo Logic", International Journal of recent Technology and Engineering (IJRTE), ISSN
[10] Anilgantala, Dasari Swathi, J Sravana, Paparao Nalajala," Automatic License Plate Detection And Character Recognition In License Plate International Journal Of Advanced Trends In Computer Science And Engineering, Vol.5, No.1, Pages : 46-50, (2016)

[11] Srihari C1,Anil Reddy G, Sruthi L,"An High Speed Two 'S Complement Multiplier Realization In Fpga", International Journal Of Advanced Trends In Computer Science And Engineering, Vol.5 , No.1, Pages : 65-69, (2016) 